europhysicsnews

2022 . Volume $53 \cdot$ number 1

Europhysics news is the magazine of the European physics community. It is owned by the European Physical Society and produced in cooperation with EDP Sciences. The staff of EDP Sciences are involved in the production of the magazine and are not responsible for editorial content. Most contributors to Europhysics news are volunteers and their work is greatly appreciated by the editors and the Editorial Advisory Board.

Europhysics news is also available online at: www.europhysicsnews.org

Editor: Els de Wolf (NL)

Email: epn.editor@eps.org - Twitter @elsdewolf1

Science Editor: Ferenc Igloi (HU)

Email: igloi.ferenc@wigner.mta.hu

Executive Editor: David Lee

Email: david.lee@eps.org

Graphic designer: Xavier de Araujo

Email: xavier.dearaujo@eps.org

Director of Publication: Agnès Henri

Editorial Advisory Board:

Richard Zeltner (Young Minds), Tracey Clarke (UK), Gonçalo Figueira (PT), Zsolt Fülöp (HU), Manuel Güdel (A), Tero Heikkilä (FI), Agnès Henri (FR), Jo Hermans (NL),

Christoph Keller (NL), Antigone Marino (IT), Arnulf Ouadt (DE),

Laurence Ramos (FR), Chris Rossel (CH),

Victor R. Velasco (SP), Sylvain Viroulet (FR)

๑ European Physical Society and EDP Sciences

\section{EPS Secretariat}

Address: EPS .6 rue des Frères Lumière

68200 Mulhouse - France

Tel: +33 $389329440 \cdot$ fax: +33 389329449

www.eps.org

Secretariat is open 09.00-12.00 / 13.30-17.30 CET except weekends and French public holidays.

\section{EDP Sciences}

Managing \& Publishing Director: Agnès Henri

Email: agnes.henri@edpsciences.org

Production Manager: Florence Anglezio

Advertising Manager: Bernadette Dufour

Email: bernadette.dufour@edpsciences.org

Address: EDP Sciences

17 avenue du Hoggar - BP 112 - PA de Courtabœuf

F-91944 Les Ulis Cedex A . France

Tel: +331691875 75 • fax: +33169288491

www.edpsciences.org

\section{Subscriptions}

Individual Members of the European Physical Society receive Europhysics news free of charge.

Members of EPS National Member Societies receive Europhysics news through their society, except members of the Institute of Physics in the United Kingdom and the German Physical Society who have access to an e-version at www.europhysicsnews.org. The following are 2021 print version subscription prices available through EDP Sciences (Prices include postal delivery cost). Institutions - European Union countries: $113 €$ (VAT not included, 20\%). Rest of the world: $136 €$ Student - European Union countries: $54.17 €$ (VAT not included, 20\%). Rest of the world: $65 €$

Contact: Europhysics News, EDP Sciences

17 avenue du Hoggar - Parc d'activités de Courtaboeuf

BP 112 - F-91944 Les Ulis CEDEX A, France

subscribers@edpsciences.org

or visit www.edpsciences.org

ISSN 0531-7479 • ISSN 1432-1092 (electronic edition)

Printer: Fabrègue · Saint-Yrieix-la-Perche, France

Legal deposit: February 2022

\section{First neutrino interaction candidates at Large Hadron Collider}

\author{
- Tomoko Ariga - Kyushu University, Nishi-ku, \\ 819-0395 Fukuoka, Japan - DOI: https://doi.org/10.1051/epn/2022101
}
The FASERv pilot detector observed the first neutrino interaction candidates at the Large Hadron Collider (LHC), opening a new avenue for studying neutrinos from current and future high-energy colliders.

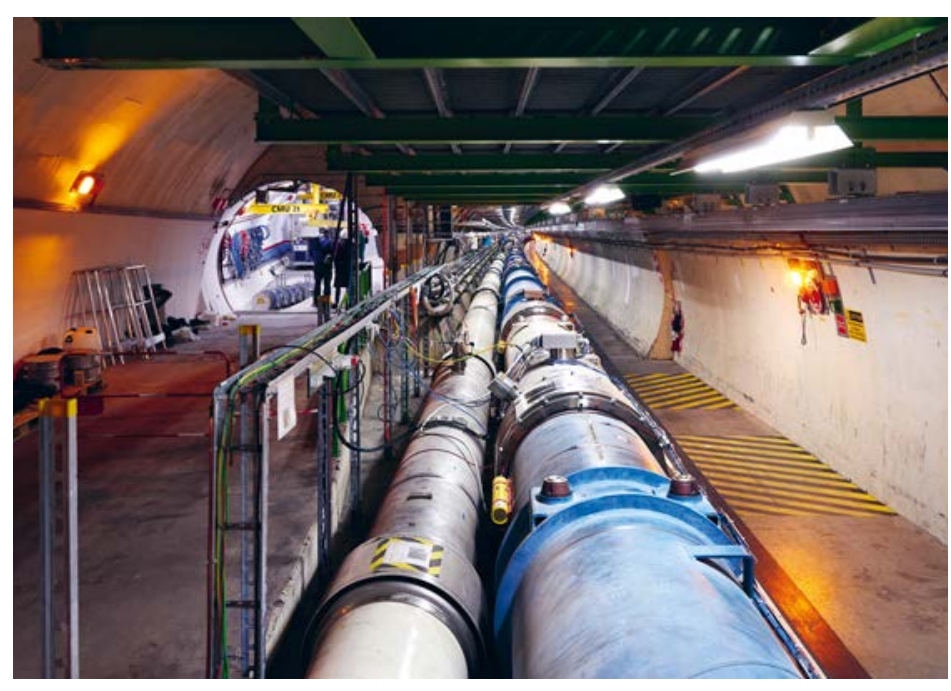

$\triangle$ FIG. 1: FASER detector installed inside tunnel shown in upper-left part of photograph.

\section{FASERv}

There has been a longstanding interest in studying neutrinos produced at the Large Hadron Collider (LHC), although collider neutrinos have not been directly detected. In Run 3 of the LHC starting from 2022, proton-proton collisions at a center-of-mass energy of $13.6 \mathrm{TeV}$ and with an expected integrated luminosity of $150 \mathrm{fb}^{-1}$ will produce a high-intensity beam of $O\left(10^{12}\right)$ neutrinos in the far-forward direction with a mean interaction energy of about $\sim 1 \mathrm{TeV}$. In the ForwArd Search ExpeRiment (FASER) [1], a neutrino detector, called FASER $v$, was designed to detect these neutrinos and examine their properties [2]. In 2021, the main FASER detector was installed $480 \mathrm{~m}$ downstream of the ATLAS interaction point inside tunnel TI12 (Figs. 1 and 2). The FASER $v$ detector, consisting of an emulsion/tungsten detector, a veto detector and an interface silicon detector, is being installed in front of it. Beam exposure and data collection will be conducted from 2022. FASER $v$ is located on the beam collision axis to maximise the interaction rate of the neutrinos of all three flavors, namely $v_{e}, v_{\mu}$, and $v_{\tau}$. This deployment allows FASER $v$ to measure the charged-current interaction cross-sections in the TeV energy range, which is currently unexplored. The FASER $v$ measurements can probe the gap between accelerator measurements $\left(E_{v}<360 \mathrm{GeV}\right)$ [3] and the IceCube 
data $\left(E_{v}>6.3 \mathrm{TeV}\right)$ [4] for muon neutrinos. For electron and tau neutrinos, the existing cross-section measurements can be extended to significantly higher energy values. In addition to the charged-current interactions, neutral-current interactions can be measured. Such measurements can provide a new limit on the nonstandard interactions of neutrinos to complement existing limits [5]. Furthermore, forward hadron production, which is poorly constrained by other LHC experiments, can be investigated using FASERv. Currently, the uncertainty in forward charm production limits the clarification of the atmospheric neutrino background to astrophysical neutrino observations using neutrino telescopes. FASERv measurements of high-energy electron neutrinos, which mainly originate from charm decays, can provide the first data on high-energy and large-rapidity charm production, providing vital data in a controlled environment for astrophysical neutrino observations.

\section{First neutrino interaction candidates}

In 2018 during LHC Run 2, a pilot run was performed in the TI18 tunnel of the LHC beamline to demonstrate neutrino detection at the LHC for the first time. The data obtained from the pilot detector were used to prove the feasibility of high-energy neutrino measurements in this experimental environment. The pilot detector was incapable of identifying muons because its depth was only $0.6 \lambda_{\text {int }}$, significantly shorter than the $8 \lambda_{\text {int }}$ of the full FASER $v$ detector for LHC Run 3. We searched for neutrino interactions by analysing

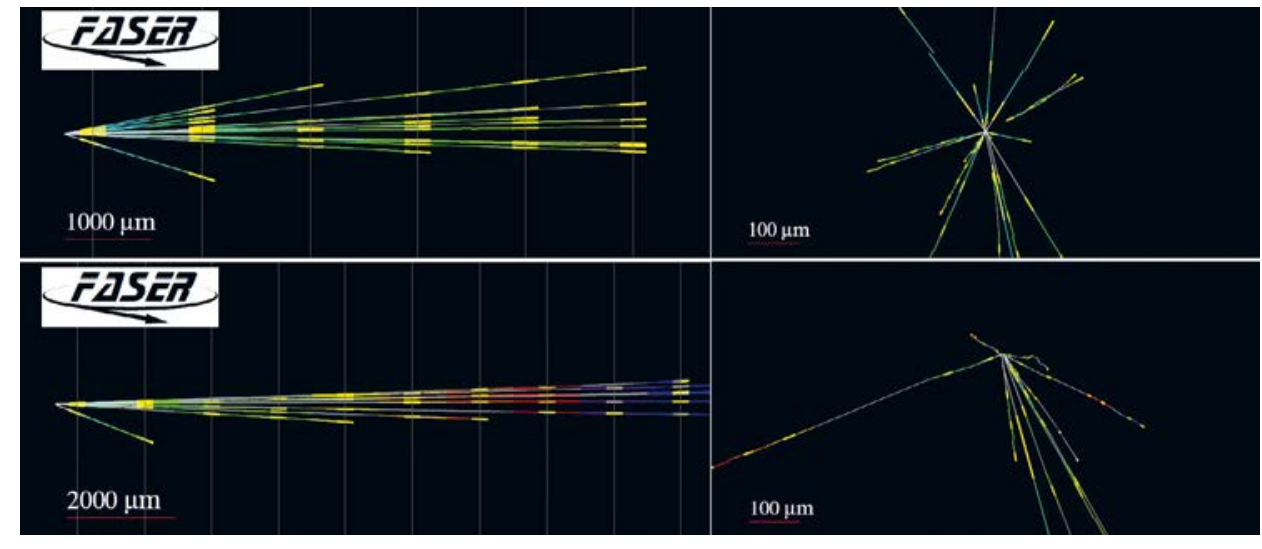

the data corresponding to $11 \mathrm{~kg}$ of the target mass. We observed the first candidate events consistent with neutrino interactions, owing to neutrinos from the LHC [6]. Fig. 3 shows two candidate events. A $2.7 \sigma$ excess of neutrino-like signals over the muon-induced background was measured. These results demonstrate the ability of FASERv to detect neutrinos at the LHC, thus paving the way for future collider neutrino experiments.

\section{Prospects}

The preparation of the FASER $v$ detector for obtaining data in LHC Run 3 is underway. With a deeper detector and lepton identification capability, FASER $v$ will perform better than the 2018 pilot detector. In 2022-2024, we expect to record about $\sim 10,000$ flavour-tagged charged-current neutrino interactions during LHC Run 3, along with neutral-current interactions. Furthermore, toward the high-luminosity LHC era, we are studying the possibility to conduct the more sensitive experiment (FASERv2) at a proposed facility known as the Forward Physics Facility [7].
\ FIG. 3: Event displays of two neutrino interaction candidate vertices [6] in $y-z$ projection longitudinal to beam direction (left) and in view transverse to beam direction (right).

v FIG. 2: Location of FASER detector.

\section{References}

[1] A. Ariga et al. (FASER Collaboration), FASER's physics reach for long-lived particles, Phys. Rev. D 99, 095011 (2019). https://journals.aps.org/prd/ abstract/10.1103/PhysRevD.99.095011

[2] H. Abreu et al. (FASER Collaboration), Detecting and studying high-energy collider neutrinos with FASER at the LHC, Eur. Phys. J. C 80, 61 (2020). https://link.springer.com/article/10.1140 1\%2Fepjcl\%2Fs10052-020-7631-5

[3] P. A. Zyla et al. (Particle Data Group Collaboration), Review of particle physics, PTEP 2020, $083 \mathrm{C01}$ (2020). https://academic.oup.com/ptep/ article/2020/8/083C01/5891211

[4] M. Aartsen et al. (IceCube Collaboration), Measurement of the multi-TeV neutrino cross section with IceCube using Earth absorption, Nature 551, 596 (2017). https://www.nature.com/articles/ nature24459

[5] A. Ismail, R.M. Abraham, F. Kling, Neutral current neutrino interactions at FASERv, Phys. Rev. D 103, 056014 (2021). https://journals.aps.org/prd/ abstract/10.1103/PhysRevD.103.056014

[6] H. Abreu et al. (FASER Collaboration), First neutrino interaction candidates at the LHC, Phys. Rev. D 104, L091101 (2021). https://journals.aps.org/prd/ abstract/10.1103/PhysRevD.104. L091101

[7] L.A. Anchordoqui et al., The Forward Physics Facility: Sites, Experiments, and Physics Potential, arXiv:2109.10905 (2021). https://arxiv.org/abs/2109.10905.

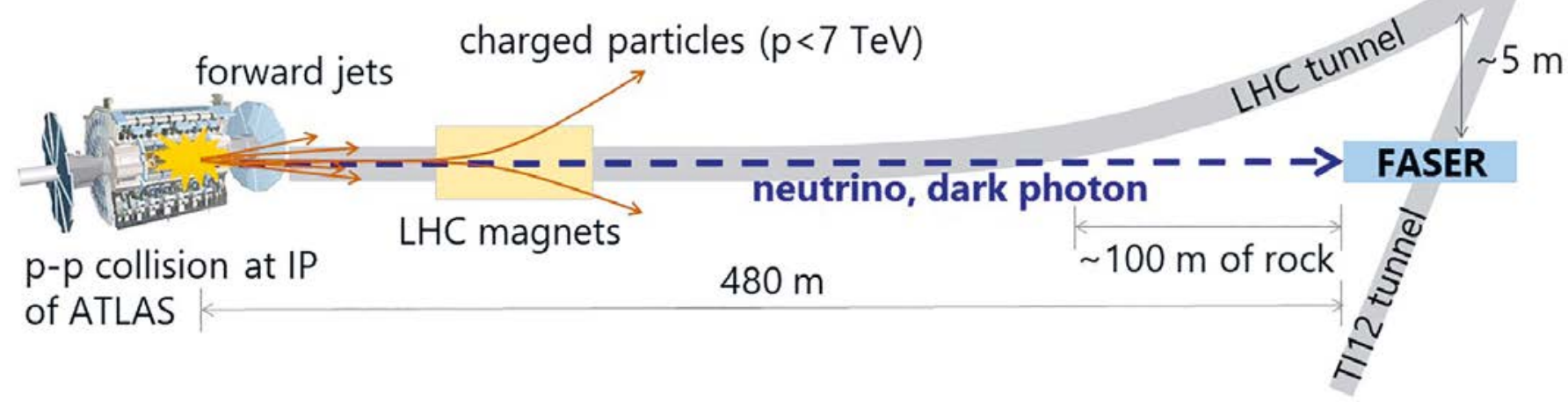

